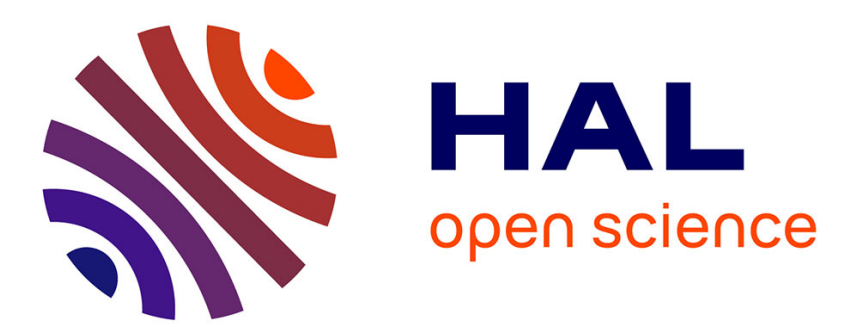

\title{
De la révolte aux libertés. L'intégration politique modèle d'un bailliage montagnard: le Briançonnais au XIV siècle
}

Anne Lemonde

\section{- To cite this version:}

Anne Lemonde. De la révolte aux libertés. L'intégration politique modèle d'un bailliage montagnard: le Briançonnais au XIV siècle. Actes de la Société des historiens médiévistes de l'enseignement supérieur public - SHMESP , 2003, 34 (1), pp.137 - 149. 10.3406/shmes.2003.1852 . hal-01936050

\section{HAL Id: hal-01936050 \\ https://hal.science/hal-01936050}

Submitted on 27 Nov 2018

HAL is a multi-disciplinary open access archive for the deposit and dissemination of scientific research documents, whether they are published or not. The documents may come from teaching and research institutions in France or abroad, or from public or private research centers.
L'archive ouverte pluridisciplinaire HAL, est destinée au dépôt et à la diffusion de documents scientifiques de niveau recherche, publiés ou non, émanant des établissements d'enseignement et de recherche français ou étrangers, des laboratoires publics ou privés. 


\section{De la révolte aux libertés. L'intégration politique modèle d'un} bailliage montagnard : le Briançonnais au XIV siècle

\section{Madame Anne Lemonde-Santamaria}

\section{Citer ce document / Cite this document :}

Lemonde-Santamaria Anne. De la révolte aux libertés. L'intégration politique modèle d'un bailliage montagnard : le Briançonnais au XIV siècle. In: Actes des congrès de la Société des historiens médiévistes de l'enseignement supérieur public, $34^{\mathrm{e}}$ congrès, Chambéry, 2003. Montagnes médiévales. pp. 137-149;

doi : https://doi.org/10.3406/shmes.2003.1852

https://www.persee.fr/doc/shmes_1261-9078_2004_act_34_1_1852

Fichier pdf généré le 31/03/2018 


\title{
De la révolte aux libertés L'intégration politique modèle d'un bailliage montagnard: le Briançonnais au XIV siècle
}

\author{
Anne LEMONDE
}

Il est de tradition, lorsque l'on s'intéresse à la genèse de la Confédération helvétique, d'effectuer un petit excursus bien plus au sud, au cœur du Dauphiné, en comparant la geste des Waldstetten dans la deuxième moitié du XIII ${ }^{c}$ siècle à celle du Briançonnais au demi-siècle suivant ${ }^{1}$ : née en 1343, la "république des escartons" (comme l'on disait autrefois) serait une réplique, moins bien réussie certes, mais une réplique tout de même, du pacte de 1291. Non que l'on ait imaginé un seul instant une influence directe de l'un sur l'autre - la distance est bien trop grande; il s'agit de deux mondes totalement étrangers : ce que l'on envisage ici, de façon tout à fait explicite, c'est une totale surdétermination de l'histoire par le cadre géographique, forme exacerbée de la surdétermination que l'on trouve sous-jacente, peu ou prou, dans toute l'histoire de ces ensembles bien plus larges que sont les principautés alpines.

Une telle problématique est extrêmement familière aux géographes ${ }^{2}$. Il existe bien des similitudes de structures économiques, sociales et politiques, évidentes et fascinantes pour l'historien, que l'on trouve toujours intérêt à rappeler. Le moment envisagé ici - la charnière du XIII siècle - est celui du renforcement du pouvoir princier alpin ; en l'occurrence, pour ce qui nous concerne, ce moment est celui d'une véritable prise en main de l'espace montagnard, dans sa totalité, par des

\footnotetext{
1. Voir notamment, J.-F. Bergier, Guillaume Tell, Paris, 1988, p. 27, 44, 304 et surtout p. 313 et suiv.

2. Beaucoup moins aux historiens, du moins en France. L'historiographie suisse et surtout italienne s'est, depuis longtemps, penchée sur cette question. Voir par exemple, les analyses de G. Castelnuovo dans "Lo spazio alpino medievale e il principato sabaudo: modelli, gerarchie, frontiere ", Archivio per l'alto Adige. Rivista di studi alpini, LXXXVIII-LXXXIX (1994-1995), p. $483-490$.
} 
princes montés, plus ou moins rapidement, de la plaine. Du point de vue des populations montagnardes, ce processus politique s'inscrit dans un processus plus large, celui d'une ouverture de plus en plus grande sur les zones de plaine et de piémont. Et même si ces espaces alpins n'ont jamais connu l'âge d'or d'un isolement total, si les routes qui les traversent les ont toujours "structurés ", désormais, à partir du XIII ${ }^{c}$ siècle, on peut dire que c'est l'espace alpin tout entier, comme territoire, qui n'existe que dans son interdépendance avec la plaine voisine.

Tout le problème est d'expliquer la différence, notamment ici la différence dans le processus d'intégration politique de l'espace montagnard et son résultat : d'un côté la réussite, l'accomplissement d'un modèle relativement neuf issu du pacte unissant les Waldstetten en 1291 ; d'un autre côté, selon l'historiographie traditionnelle, le demiéchec de ce modèle, en Briançonnais ${ }^{3}$. En général, la gémellité des deux modèles n'est pas mise en doute, fruit de ces conditions géographiques surdéterminantes, précisément. Seuls les résultats sont comparés : leur écart, entre réussite et demi-échec, étant attribué aux quelques dissemblances, de structures toujours, que l'on peut pointer. Une première réflexion, ponctuelle, voudrait être ébauchée ici. Le cas des Waldstetten est bien connu et a été revisité récemment; c'est donc sur l'exemple des escartons du Briançonnais que l'on se penchera plus spécialement.

Tout entier situé au-dessus de 1000 mètres d'altitude, cerné de toute part de sommets de plus de 3000 mètres, le bailliage de Briançonnais est bien un ensemble montagnard par excellence : la similitude avec les Waldstetten, de même superficie, est, de ce point de vue, incontestable. C'est un lieu commun toujours nécessaire que de rappeler l'âpreté de ces montagnes, qui vous raye de la carte des hameaux entiers sans crier gare à la moindre intempérie tant soit peu violente (et fréquente ici du fait de l'influence méditerranéenne), où la saison végétative est si courte que le moindre accident climatique peut être fatal. Ces montagnes sont pourtant bien peuplées, parce que, comme presque toujours, il est possible de les traverser. Avant la grande peste, on y compte à peu près 30000 habitants, soit le même ordre de grandeur que dans les Waldstetten ${ }^{4}$. La grande différence avec les vallées suisses réside

3. Deux études demeurent les références obligées sur les escartons : A. Fauché-Prunelle, Essai sur les anciennes institutions autonomes ou populaires des Alpes cottiennes-briançonnaises, 2 vol., Grenoble-Paris, 1856 ; P. Vaillant, Les libertés des communautés dauphinoises, Paris, 1951 et "Les origines d'une libre confédération de vallées: les libertés des communautés briançonnaises au $\mathrm{XII}^{2}$ siècle ", Bibliothèque de l'École des Chartes, 128 (1967) p. 301-348. Plus récemment, H. Falque-Vert a consacré une analyse nourrie à la question dans Les hommes et la montagne en Dauphiné au XIIf siècle, Grenoble, 1998, p. 439-449.

4. Ibid., p. 17-49. 
en fait dans le rapport au monde urbain, et c'est elle qui est en général avancée pour expliquer le demi-échec des escartons ${ }^{5}$. Dans le cas suisse, la grande ville est proche, même si elle est en dehors : c'est Berne. En Briançonnais, le monde urbain est beaucoup plus éloigné et ses liens sont assez lâches avec ces montagnes. Certes, il existe ici un grand axe, beaucoup plus ancien que celui du Gothard, celui de la Durance vers le Montgenèvre, puis Césanne, Oulx et la Doire Ripaire, grand axe qui vit se développer dans l'antiquité les cités de Gap et d'Embrun, elles aussi situées aux portes du Briançonnais. Mais au XIII siècle, elles se sont quelque peu assoupies, au point que l'on peut à peine parler de villes à leur sujet. Le Montgenèvre n'a pas le succès du Saint-Gothard, il s'en faut de beaucoup.

En amont, cependant, un autre bourg entre dans une phase de grande prospérité : il s'agit de Briançon qui connut un premier apogée au temps du séjour des papes à Avignon. De grands historiens en ont retracé la destinée, je ne m'y attarderai pas ${ }^{6}$. Quoi qu'il en soit, le monde véritablement urbain n'est pas monté si haut : située au débouché naturel des "montagnes", Briançon est une place de foires actives à partir du XIII siècle, animées par de dynamiques marchands, mais où le commerce est exclusivement lié à l'élevage. Hors du grand axe Durance-Doire Ripaire et sa petite ramification vers le Lautaret, le Briançonnais est essentiellement constitué de vallées plus ou moins en culs-de-sac qui conduisent précisément aux alpages et à des cols inaccessibles sept ou huit mois par an mais indispensables aux communications locales : or ces vallées sont véritablement la chair de l'espace, du territoire briançonnais.

Dès la fin du XI siècle, le Briançonnais peut être considéré comme le point d'achèvement de cette première "aire de routes" rapidement assemblée par les Guigues, ancêtres des dauphins, et légitimée par les empereurs à Briançon et Césane, pour l'essentiel' ${ }^{7}$. Hors de la route, dans chacune des vallées en culs-de-sac évoquées, les seigneurs d'Albon sont presque totalement absents. Bien présente en revanche, une noblesse prolifique, majoritairement pauvre, mais très hiérarchisée, tant et si bien que quelques grandes familles sortent réellement du lot : au XIII ${ }^{e}$ siècle, on

5. Notamment par les auteurs cités supra.

6. H. Falque-Vert, Les hommes et la montagne..., op. cit., p. 439-449. Voir également, T. Sclafert, Le Haut Dauphiné au Moyen âge, Paris, 1926 et R. Chanaud, "Le Briançonnais aux XIV et XV siècles. Aspects de la vie économique ", PTEC, 1974, p. 37-44.

7. G. Giordanengo, Le droit féodal dans les pays de droit écrit, Rome, 1988 et G. Sergi, Potere e territorio lungo la strada di Francia. Da Chambéry a Torino fra X e XIII secolo, Naples, 1981, notamment p. 54-57 et p. 139-154; G. Letonnelier, "Essai sur les origines des châtelains et mandements en Dauphiné ", Annales de l'Université de Grenoble, 1 (1924), p. 1-40 et 2 (1925), p. 211-230. 
les repère sous les patronymes d'Auruce, de Bermond, de Névache ou de Bardonnèche. Petits ou grands, ces nobles exercent un pouvoir qui est vécu comme tyrannique par les rustres montagnards : l'harmonie sociale qui règne dans les Waldstetten n'a donc point cours ici. Pourtant, tout comme leurs homologues des Waldstetten, jamais les Briançonnais ne se laissèrent asservir par leurs maîtres ${ }^{8}$.

Le commencement du XIII siècle marque, aussi bien dans les montagnes suisses que dauphinoises, le début d'un processus d'ouverture de tout l'espace montagnard, à tout point de vue (nous avons évoqué les alpages). Au même moment, selon l'historiographie traditionnelle, les descendants des Guigues opèrent une vigoureuse reprise en main politique, sur toutes les terres qu'ils détiennent, y compris dans l'avant-pays ; après la phase "féodale", la phase du prince souverain". Plus encore en Briançonnais qu'ailleurs, selon ce schéma, le prince est nécessairement en lutte contre la noblesse, rogne ses droits, et cherche des appuis dans les communautés de rustres qui reçoivent de sa main leurs premières chartes de libertés quand elles sont assez fortunées pour se les offrir (Exilles 1243, Briançon 1244$)^{10}$. Les grandes familles nobles renâclent, évidemment, se révoltent les unes après les autres, les Auruce et les Bermond au XIII ${ }^{e}$ siècle, les Bardonnèche et les Névache autour de $1330^{11}$, pour être finalement, selon cette historiographie, inévitablement brisées et laminées par le pouvoir delphinal. Dans ces conditions, les rustres tirent de mieux en mieux leur épingle du jeu et en viennent à conduire le dauphin beaucoup plus loin qu'il ne l'aurait voulu ${ }^{12}$. C'est ainsi qu'en 1343 ils obtiennent enfin la concession de libertés extrêmement larges, à travers la grande charte fondatrice des escartons. L'historiographie traditionnelle y voit le fruit d'un véritable rapport de force, dans lequel les paysans l'auraient emporté. Elle insiste également, à bon droit me semble-t-il, sur la nouveauté que représente cette charte : en fait, les innovations y sont multiples, mais au fondement de toutes se trouve la reconnaissance définitive des communautés de paroisses, auxquelles sont confiés des pouvoirs assez vastes, similitude essentielle avec la confédération des Waldstetten ${ }^{13}$. Sauf que dans ce dernier cas, le prince n'est pas intervenu

8. H. Falque-Vert, Les hommes et la montagne..., op. cit., passim.

9. Voir notamment les analyses de P. Vaillant, Les libertés..., op. cit., p. 262 et suiv., et "Les origines...", art. cit., p. 315.

10. Ibid.

11. H. Falque-Vert, Les hommes et la montagne..., op. cit., p. 361-436.

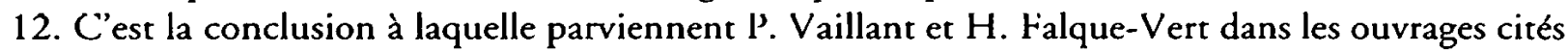
supra.

13. Voir notamment les analyses très fines au sujet des communautés d'H. Falque-Vert, Les hommes et la montagne..., op. cit., passim et plus particulièrement p. 439-449. 
et que les pouvoirs que s'attribuent les communautés vont bien au-delàa ${ }^{14}$. En Briançonnais, c'est seulement la basse justice (les peines jusqu'à 10 sous) qui est confiée aux bénéficiaires des libertés et, surtout, la gestion de l'ensemble des redevances autrefois perçues par le prince ${ }^{15}$. Dorénavant, ce sont les communautés qui vont "escartonner ", répartir donc, les tailles et les cens, à leur convenance. Selon l'historiographie traditionnelle, l'absence de ville-relais à proximité, jointe à l'arrivée des Valois en Dauphiné en 1349 aurait empêché que cette "république" s'épanouisse complètement.

Je suggère donc une relecture de cet enchaînement. De fait, on observe bien les débuts d'une mutation fondamentale de la nature du pouvoir des comtes d'Albon avec Guigues André (1192-1236), tige de la dynastie de Bourgogne en Dauphiné, inventeur du titre delphinal, autant dire du titre princier. Lui et son fils Guigues VII (1236-1270) choisirent d'asseoir leur pouvoir sur des bases territoriales, nouveauté et non reconquête sur une féodalité mythique, à mon sens ${ }^{16}$. Le territoire ainsi créé reste discontinu, mité de toutes parts, mais il a pour caractéristique essentielle une forme d'homogénéité spatiale. Les modalités de sa construction sont les mêmes en Briançonnais que sur les autres terres delphinales : des châtelains et des mistraux sont installés sur des châtellenies et des mistralies qui leur sont inféodées; les nobles, petits et grands, sont invités à prêter hommage au prince et à reprendre en fief un grand nombre de leurs terres allodiales et, de plus en plus, les plus pauvres acceptent de vendre une part de leur seigneurie au dauphin; les rustres, eux, sont donc de plus en plus souvent soumis à la seigneurie delphinale, notamment par le biais de ces co-seigneuries. Il est évident que la grande misère de la majorité de la noblesse fut ici une aubaine pour le dauphin : le Briançonnais est la zone la plus soumise, et de loin avec l'Oisans, de tout l'ensemble dauphinois que l'on ne saurait encore qualifier de principauté. Sans doute le fait qu'il soit montagnard y est-il

14. L'analyse la plus récente de la charte suisse est celle de J.-F. Bergier, Guillaume Tell..., op. cit., p. 336 et suiv.

15. La charte a été éditée par A. Fauché-Prunelle, Essai sur les anciennes institutions..., op. cit., vol. 2, p. 347-369. Les deux passages clés sont les suivants: Quod quelibet universitas dicte bayllivie blada ipsi domino Dalphino debita deinceps habeat et sibi retineat universaliter et singulariter (p. 352), et: etiam quod ipsi syndici, durante eorum administrationis tempore, pro predictis et infrascriptis rebus debitis domino Dalphino..., autoritate tamen dalphinali, et tamquam dalphinales maignerii in hoc casu, possint quibuscumque penas imponere usque ad quinque vel decem solidos... (p. 356).

16. Nous avons déjà développé tous ces aspects dans notre thèse, à laquelle nous nous permettons de renvoyer : A. Lemonde, De la principauté delphinale à la principauté royale. Structures et pouvoir en Dauphiné au XIV siècle, Grenoble, 2000, vol. 1, chapitre introductif. Voir également H. FalqueVert, Les hommes et la montagne..., op. cit., p. 361-436. 
pour beaucoup; mais le déterminisme est ici inversé par rapport aux Waldstetten fondamentalement libres. Cette nouvelle seigneurie delphinale bénéficie d'une gestion impeccable et inédite; de grandes enquêtes sont organisées entre 1250 et 1265 que l'on désigne habituellement sous le nom du registre qui les contient : le Probus ${ }^{17}$. Elles rassemblent notamment toutes les reconnaissances des tenanciers delphinaux. Et bien entendu, le Briançonnais y tient la part du lion; rançon du succès, le dauphin est partout, jusque dans les plus hautes vallées en cul-de-sac, mais toujours associé à d'autres, dans une imbrication phénoménale de droits avec la petite noblesse. Seule une haute vallée échappe à sa seigneurie, sauf l'hommage : la vallée de Bardonnèche solidement dirigée par la grande famille. Ici, les Bardonnèche forment un clan puissant (par le nombre plus que par la richesse au milieu du XIII siècle) et exercent un pouvoir quasi souverain : un potestat est leur officier dont on ne trouve pas l'équivalent ailleurs en Dauphiné, et un Conseil des co-seigneurs dirigé par un recteur se présente comme un embryon institutionnel ; ici, la justice delphinale n'a absolument pas cours ${ }^{18}$.

En Briançonnais plus qu'ailleurs encore, cette prise en main territoriale s'effectue bien davantage en association avec la noblesse qu'en opposition avec elle, même si, de fait, la superioritas du dauphin se trouve systématiquement confortée et reconnue par les nobles par une féodalisation des relations de l'un avec les autres. Ceux-ci y trouvent leur compte: les offices de châtelains et de mistraux leur reviennent et nombre de bourses ont dû se regarnir lors des achats de droits seigneuriaux $^{19}$. Les paysans ont beau dénoncer la tyrannie des reges, des challains - les noms par lesquels ils désignent les petits nobles - le dauphin ne fait finalement qu'y ajouter la sienne, plus stricte encore, puisqu'elle implique la diffusion du statut "d'homme lige " du dauphin, un statut fort proche de la servitude, qui s'assortit d'un hommage plébéien humiliant, avec baiser sur les pouces du seigneur. Les libertés conférées aux communautés

17. L'enquête est au cœur de toute l'étude de H. Falque-Vert, Les hommes et la montagne..., op. cit., passim.

18. C'est la charte concédée par les co-seigneurs en 1330 qui permet le mieux de comprendre cette emprise des Bardonnèche sur la haute vallée. Elle est éditée par A. Fauché, Essai sur les anciennes institutions..., op. cit., vol. 2, p. 374-388. Sur le potestat: Item, promisit ut supra praedictis sindicis et notariis stipulantibus ut supra quod ipse ponet, in dictis locis, rectorem, potestatem seu judicem, omni suspicione carentem, qui regat terram et villas predictas in villa Bardonnechiae...(p. 376). Sur le Conseil : Item pactum extitit... quod capitulum Bardonechiae observetur una cum praedictis conventionibus... tamen si dictum capitulum in aliquo casu correctione vel declaratione indigeret, pracdicta correctio et declaratio fiat in consilio dominorum Bardonechiae...(p. 382).

19. Sur cette alliance objective, fondamentale, voir A. Lemonde, De la principauté delphinale..., op. cit. 
L'intégration politique modèle d'un bailliage montagnard 143

les plus riches n'enlèvent donc rien aux nobles du lieu : en échange de droits d'introge conséquents, elles se contentent de transformer ce statut d'homme lige en celui, ô combien plus honorable en Dauphiné, d'affranchi, d'abonner la taille et de reconnaître l'existence de la communauté concernée $e^{20}$. La grande noblesse briançonnaise, quant à elle, n'est pas plus pourchassée que la petite : elle trouve une place à sa mesure dans le nouveau Conseil du dauphin, à l'exemple d'Obert Auruce, le maréchal ${ }^{21}$. Celui-ci connut la disgrâce non pour s'être révolté en Briançonnais, mais à l'issue d'une lutte politique au sommet.

Avec les dauphins de la troisième race (1282-1349), la territorialisation du pouvoir delphinal entre dans une nouvelle phase ${ }^{22}$. Désormais la continuité est de mise et son pilier est un tout nouvel ordre administratif : les châtelains deviennent des officiers gagés et contrôlés quasi annuellement et les châtellenies sont regroupées dans des bailliages, nom uniforme donné aux vieilles circonscriptions postcarolingiennes. C'est à ce moment-là qu'apparaît le nom de Dauphiné et que le dauphin s'impose, partout, comme suprême juge. Et le Briançonnais n'est plus le fer de lance de la nouvelle souveraineté delphinale: d'autres bailliages bien plus riches rapportent plus et retiennent davantage l'attention du prince. D'une certaine façon, le Briançonnais pose même problème au dauphin qui peine à l'administrer à la mode nouvelle: les droits seigneuriaux y sont tellement imbriqués !

En 1338, Humbert II lança dans toute sa principauté une grande campagne de renouvellement des reconnaissances de droits seigneuriaux delphinaux. Les communautés du Briançonnais refusèrent de s'y soumettre, signant par là, selon l'historiographie traditionnelle, leur rébellion contre le pouvoir delphinal ${ }^{23}$. Le texte de la grande charte de 1343, qui

20. P. Vaillant, Les libertés..., op. cit., et "Les origines... ", art. cit., passim. Sur le statut de franc: V. Chomel, "Francs" et "rustiques" dans la seigneurie dauphinoise au temps des affranchissements ", Bulletin philologique et historique, 90 (1965), p. 285-308.

21. Sur le destin du maréchal, voir $\mathrm{H}$. Falque-Vert, Les hommes et la montagne..., op. cit., p. 363368.

22. A. Lemonde, De la principauté..., op. cit., chapitres 1, 2 et 3.

23. Ces campagnes de reconnaissances ont été étudiées par nos soins dans notre thèse (voir supra note 22). Le refus du Briançonnais est mentionné notamment dans le préambule de la charte de 1343 : cf. A. Fauché, Essai sur les anciennes institutions..., op. cit., vol. 2, p. 347 : Ordinatum extiterit fieri recognitiones et insuiri dalphinalia jura per universum Dalphinatum... [et] suborta fuerit materia questionis cum universitatibus dicte bayllivie et singularibus personis earumdem... super eo quod dicte universitates requisite per dictos commissarios universaliter, et etiam singulares persone recognoscere particulariter et distincte dalphinalia jura, ipsis... asserentibus hoc non posse facere, nec facere debere, nisi modo et forma quibus circa quatuor viginti anni sunt elapsi, recognita ipsa jura dalphinalia, ut asseritur, extiterunt, et in libris seu cartulariis dalphinalis curie continetur... On le 
évoque cet épisode, ne parle cependant pas de rébellion, et l'on ne trouve nulle part ailleurs mention de quelconques violences de la part de ces communautés, sauf outre-mont, j'y reviendrai. Le préambule se contente de dire que les habitants veulent bien se soumettre à l'obligation de reconnaissance, mais selon les modalités du Probus, qui n'ont pas été mises à jour depuis cette date, insistent-ils; et ils arguent plus loin de leur simplicitas pour refuser une telle mise à jour ${ }^{24}$. Derrière l'argument, c'est bien sûr la complexité, l'inextricable enchevêtrement de droits qu'il faut lire : il n'y a pas vraiment là d'insoumission, mais bien plutôt du réalisme (même si les communautés ont bien compris où était leur intérêt et que la rusticité est avancée avec beaucoup de malice !).

Comment ne pas voir alors dans la concession delphinale de 1343 le choix de la rationalité et de l'efficacité administratives ? Outre des droits d'introge conséquents (12 000 florins), le dauphin était désormais assuré de toucher à la chandeleur en une fois, chaque année, l'équivalent de ce que ses officiers devaient lever en déployant des trésors d'énergie ${ }^{25}$. Certes, cela impliquait en échange de renoncer à gouverner au jour le jour ces populations, comme un seigneur à l'ancienne ; cela impliquait en fait d'affranchir la totalité des communautés impliquées par la charte ${ }^{26}$ : et il semble bien que ce fut Humbert II qui en décida ainsi, et non les communautés qui le lui arrachèrent. La souveraineté du prince, de son juge associé aux châtelains se trouvait soigneusement mentionnée dans des exceptions systématiques aux libertés concédées. C'est ainsi que les communautés de paroisses sont autorisées à s'assembler à son de trompe, mais seulement pour des besognes licites, en présence du châtelain et après avoir prêté serment à ce dernier ${ }^{27}$; les devoirs de guet sont abolis,

voit, la mémoire, en la matière, est longue et on y lit, a posteriori, l'importance cruciale du Probus, fondatrice à plus d'un titre, pour les paysans du Briançonnais.

24. A. Fauché, Essai sur les anciennes institutions..., op. cit., vol. 2, p. 353 : Multi possent incidere in labirintum ex ignorantia, simplicitate vel rusticitate...

25. A. Fauché, Essai sur les anciennes institutions..., op. cit., vol. 2, p. 352 : la taxe de la chandeleur sera évaluée en faisant la moyenne du rapport des cens depuis 20 ans (vicessima pars dicte grosse summe annorum viginti solvere annis singulis infesto predicto domino Dalphino supra dicto), et ibid., p. 366: Concesserunt, dederunt et expresse donaverunt prefato domino Humberto dalphino... duodecim milia florenorum auri justi et legalis ponderis, semel...

26. En conférant donc le statut de "franc" évoqué plus haut à l'ensemble des communautés contractantes (voir supra note 20). Le passage clé de la charte, concernant le statut des hommes est le suivant : Statuit et concessit quod omnes homines predicti... ex nunc inantea in perpetuum, franchi atque burgenses nuncupentur, et deinceps prestare babeant hornagia evrurn osculurdo dominum dalphinum... in anulo vel dorso manus sue, sicuti franchi.... A. Fauché, Essai sur les anciennes institutions..., op. cit., vol. 2, p. 364.

27. Ibid., p. 356. 
sauf en cas de nécessité ${ }^{28}$; le dauphin ne conduira plus d'enquêtes ex officio nisi in casibus a jure permissis... ${ }^{29}$

Ses sujets affranchis, ses fidèles, se plaît-il à répéter dans la charte, seraient désormais liés à lui par un lien politique de type contractuel, que l'on trouvait déjà dans les chartes particulières les plus tardives concédées aux communautés, mais qui se voyait ici généralisé. Généralisé à un territoire bien défini, délimité, selon les nouvelles normes progressivement mises en place depuis le temps du grand-père d'Humbert II, Humbert I ${ }^{\text {er }}$ : le territoire du bailliage. Car, preuve ultime du fait que c'est bien Humbert II qui offrit ces libertés aux communautés et non ces dernières qui les lui arrachèrent, le territoire sur lequel s'exercent ces libertés est bien celui du bailliage, sans cesse mentionnés ${ }^{30}$ Le terme luimême d'escartons n'apparaît que l'année suivante lorsqu'il fallut effectuer la première répartition entre les communautés, mais il n'est pas utilisé dans la charte ${ }^{3 P}$.

Mais alors ne s'agissait-il pas pour le dauphin, ce faisant, d'achever d'écraser définitivement la grande noblesse féodale chère à l'historiographie traditionnelle? Car, de fait, une révolte fameuse (et unique en son genre, en Dauphiné) agita le Briançonnais une décennie auparavant, entre 1328 et 1332 , encadrée, toujours selon l'historiographie traditionnelle, par François de Bardonnèche, alors sans conteste le plus puissant des nobles de la région ${ }^{32}$. Notons tout d'abord que cette agitation ne toucha que l'outre-mont, et encore pour partie seulement (Bardonnèche, Exilles, Césane, et la vallée de Bellin), ainsi que la vallée de Névache. Autrement dit, la zone frontalière avec la Savoie, au pire moment de la guerre qui opposait les deux princes. La chronologie mérite d'être affinée. En 1328, en effet, les communautés de Césane, d'Exilles et de Névache se soulèvent contre le pouvoir delphinal, soutenues en cela par Obert Auruce, descendant sans doute du maréchal. Le juge Raymond Chabert,

28. Ibid., p. 361.

29. Ibid., p. 357.

30. Ibid., p. 347 (première mention) : Ad que peragenda [i.e. faire de nouvelles reconnaissances] idem dominus Dalphinus certos in bayllivia Brianczonesii commissarios deputavit... ; et l'on apprend incidemment que c'est le bailliage de Briançonnais qui est censé bénéficier des libertés (et non la seule mythique "principauté " de Briançonnais, inventée au XI' siècle, qui ne comprend en fait que les châtellenies de la Haute-Durance, en amont d'Embrun) : Dicentibus insuper et asserentibus prefatis universitatibus et ipsarum universitatum singularibus personis gabellam et gabellas que in dicto bayllivatu pro dalphinali parte exiguntur non debere exigi, nec etiam levari precipue a personis et incolis dicti bayllivatu..., A. Fauché, Essai sur les anciennes institutions..., op. cit., vol. 2, p. 348.

31. H. Falque-Vert, Les hommes et la montagne..., op. cit., p. 439-449.

32. Voir notamment P. Vaillant, "Les origines...", art. cit., p. 316 et suiv. Je remercie ici chaleureusement le Professeur Michael Gelting qui m’a donné de très précieux renseignements sur la réaction savoyarde, côté Maurienne, à la révolte. 
qui tentait de tenir les assises annuelles à Césane, est insulté, le procureur, Jean d'Argentière, est quant à lui molesté ${ }^{33}$. Pendant deux ans au moins, ils n'osèrent plus passer le Montgenèvre ${ }^{34}$. La même année 1328, la communauté des "populaires" de Bardonnèche se soulève elle aussi : non pas contre le dauphin, dont la souveraineté est tout à fait nominale ici, mais contre les co-seigneurs de Bardonnèche précisément ${ }^{35}$. Enfin, en 1328 toujours, la même communauté d'Exilles livra au comte de Savoie son propre châtelain, qui n'était autre que Hugues de Bardonnèche, cousin du précédent... ${ }^{36}$ Rien de tout cela ne démontre l'insoumission des seigneurs de Bardonnèche, au contraire. Tout au plus doit-on y voir l'expression de l'agitation endémique d'une population paysanne qui supporte de moins en moins ses seigneurs tyranneaux et le pouvoir d'un prince bien peu présent mais si exigeant. En Maurienne, on observe une agitation concomitante qui a pu faire tâche d'huile et, d'autre part, la guerre n'a pu qu'encourager la population à profiter de la situation (si le comte de Savoie n'a pas lui-même soufflé sur les braises) ${ }^{37}$. Jusqu'en 1330 au moins, les Bardonnèche sont d'une fidélité exemplaire au dauphin. Et au premier chef François, qui est devenu au fil du temps un très puissant seigneur. Il fait partie du Conseil delphinal, prête de l'argent au dauphin à plusieurs reprises, combat à ses côtés à Varey en 1325 et comme

33. Une grande enquête, conduite par des commissaires diligentés par le dauphin, fut entamée contre ces révoltés, en 1330 : voir notamment $A D$ Isère, B 3705: $f^{\circ} 145$ un des témoins de Césane, Jean Girard, raconte: Item, interrogatus si Johannes de Argenteria procurator domini dalphini et Johannes Maurelli mistralis Cesane fuerunt maletractati per dictam communitatem Cesane... dixit quod sic et quod dicti procurator et mistralis fuerunt butati ante et retro hinc et inde et quod vituperati pro eo quod volebant pygnorare in dicta villa. ( $\left.f^{\circ} 161\right)$ Les populares de Césane et Sauze de Césane sont accusés de congrégation illicite au nombre de 50 environ, ligue illicite contre le dauphin et injures contre des officiers delphinaux, après avoir élu Obert Auruce à leur tête au cours d'une réunion dans un pré au-dessus de chez lui "où l'on mangea deux moutons". L'agitation se retrouve à Exilles et Névache ( $f^{\circ} 167$, "congrégation " illicite à Exilles et $f^{\circ} 177$, violences à Névache).

34. Le compte de châtellenie de Briançon de 1330 mentionne ainsi : officiales domini non audebant ibi [outre-mont] esse propter dissensionem Terre (AD Isère, 8 B $616 \mathrm{f}^{\circ}$ 50v).

35. La révolte est mentionnée notamment au préambule de la charte concédée par les Bardonnèche à la communauté du lieu en 1330 : Fauché, Essai sur les anciennes institutions..., op. cit., vol. 2, p. 374 : Cum questiones et discordiae variae et diversae verterentur et essent inter nobilem virum dominum Franciscum de Bardonechia... et sindicos... parochiarum Bardonechiae. La discorde n'est certes pas nécessairement révolte, mais on apprend à la fin de la charte que François accorde une amnistie totale aux habitants, propter aliquem excessum... perpetratum (ibid., p. 386). 36. La référence m'est fournie par M. Gelting: AD Savoie, SA 15994, compte de châtellenie de Maurienne de cette année-là : Recepit a Vicencio Sesterii, quia inuasit castellanum quum ducebat dominum Hugonem de Bardonechia, XVI d. tur. gross. tur. [sic]... librate, exploratores : pluribus et diuersis exploratoribus tramissis in terram Brianczonis... ante captionem domini Hugonis de Bardoneschin.

37. Voir supra note 36. 
beaucoup des membres de sa famille occupe souvent l'office de bailli de Briançonnais au cours de ces années-là ${ }^{38}$. Lui-même victime de la révolte de 1328, il n'en est évidemment pas l'instigateur.

Pourtant, quatre ans plus tard, sans doute en octobre ou novembre 1332, il trahit le dauphin et prête hommage au comte de Savoie, entrânnant avec lui un noble de Névache, Aragon, et une partie des communautés de Bardonnèche, Exilles, Césane et surtout Bellin, qui semble avoir été la plus engagée ${ }^{39}$. Fort de ce soutien, le comte lance une grande attaque en novembre 1332, mais le dauphin riposte efficacement, bien épaulé par la majorité de la population qui lui est finalement restée fidèle. C'est le seul cas de trahison que l'on rencontre durant cette période en Dauphiné ; c'est le seul cas aussi qui valut à un grand noble criminel une sentence d'une exceptionnelle duretét ${ }^{40}$. En 1345, il fut finalement précipité pieds et poings liés dans le Rhône ${ }^{41}$. La répression ordonnée par Humbert II fut sans pitié et immédiate : en 1334, Aragon de Névache eut la tête tranchée et ils furent plusieurs dizaines de ses acolytes à être pendus avant $1335^{42}$. Les hommes de Bellin qui s'étaient associés durent prêter hommage en baisant les pieds du prince dès 1334 également, ce qui était plus humiliant encore que d'embrasser ses pouces $^{43}$. Enfin, ajoutons que ces paroisses, où une part des populations avait trahi, ne furent pas d'emblée incluses dans la charte de 1343 : leur

38. A. Lemonde, De la principauté..., op. cit., p. 244-245.

39. En fair, le dauphin, Guigues VIII, devait sentir un certain flottement dès le début de l'année : il se rend sur place et conclut un "pacte " avec François et ses alliés au mois de juin 1332 où ils déclarent renoncer à toutes les gardes conclues avec le comte de Savoie (AD Isère, B 2611 f $^{\circ} 147$ $149,152-159$ et $f^{\circ} 161$ pour Bardonnèche, $f^{\circ} 195$ pour Césane) : des liens sans doute assez anciens avaient donc déjà été renforcés à ce moment-là. On apprend la trahison des autres communautés a posteriori, à travers le récit des poursuites et des sentences. Pour les gardes, voir AD Savoie (références fournies par M. Gelting), SA 15999, compte de la châtellenie de Maurienne pour l'exercice 1332-1333 et 1333-1334: mention de la garde payée par les hommes de Bardonnèche au châtelain (Item, reddit computum quod recepit a communitate parrochie de Bardoneschia de garda per annum... a communitate parrocie Richamolarii pro eodem... a communitate parrochie Beularii pro eodem...). Voir un résumé, parfois fantaisiste de l'affaire dans N. Chorier, Histoire générale de Dauphiné, Grenoble, 1661-1672, vol. 2, p. 255, 264-271 et 315 , ainsi que M. de Valbonnais, Histoire de Dauphiné et des princes qui ont porté le nom de Dauphin, particulièrement ceux de la troisième race, Genève, 1722, vol. 1, p. 303-312.

40. Retranscrite dans M. de Valbonnais, Histoire de Dauphiné..., op. cit., vol. 2, p. 257-258.

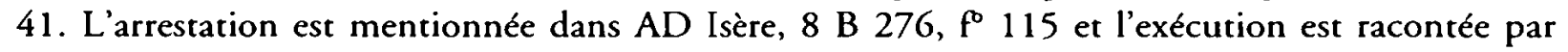
Henri de Villars dans une lettre qu'il envoya au dauphin en 1346, U. Chevalier, Choix de documents inédits sur le Dauphiné, publiés par le Bulletin de la Société statistique de l'Isère, 3' série, 6 (1874), p. 107-116.

42. AD Isère, comptes de châtellenies du Briançonnais (Briançon, Césane, Oulx, Valcluson, Bardonnèche et Château-Dauphin) : $8 \mathrm{~B} 29,8 \mathrm{~B} 30$.

43. AD Isère, B 2962, f 507, hommage au nouveau dauphin Humbert II, osculando eum in pedibus ex sentencia per ipsum dominuùm lata quia ad hoc quodammodo rebelles se pretenderant... 
adhésion est bien explicitement prévue mais non encore effective ${ }^{44}$. Pourtant, le temps de la répression était passé depuis longtemps : il fallait seulement laisser s'écouler quelques années pour parvenir à une complète réconciliation. Et pour cela, y avait-il meilleur cadre que cette grande charte qui libérait tout en soumettant à un pouvoir sans faille, bien établi derrière des frontières enfin sûres avec le monde extérieur? La trahison brutale de François de Bardonnèche reste cependant un mystère : à part une population agitée de façon endémique, qu'il put rallier pour partie en lui concédant une charte de franchises exceptionnellement libérale, il n'avait presque aucun soutien en Dauphiné. La chronique de Nicolas Chorier au XVII siècle évoque une sombre histoire de rapt de la fille de François par Guigues VIII ${ }^{45}$. On n'en trouve aucune allusion dans les sources contemporaines... On se contentera dès lors d'invoquer un désaccord, au sommet, entre le dauphin et ce grand personnage; désaccord politique (à une époque où le dauphin semble partisan d'une guerre à outrance, ce n'est pas impossible, car cette position était discutable) ou désaccord personnel, difficile de trancher. En tout cas, François de Bardonnèche fut brisé, mais non point sa famille, qui ne semble pas l'avoir suivi ${ }^{46}$. Le dauphin prit seulement le soin d'éparpiller ses biens dans tout le Dauphiné pour tenir enfin la presque-totalité des terres briançonnaises d'outre-mont en direct ${ }^{47}$.

De la similitude avec les Waldstetten et donc d'une certaine forme de déterminisme montagnard, on ne doit retenir à mon sens que la force des communautés paysannes. C'est tout à fait loin d'être négligeable, soulignons-le. Mais le résultat politique en fur, en Briançonnais, quelque chose de presque opposé à la première Confédération helvétique. Ici, les communautés accèdent à une existence politique autonome, là, le prince s'appuie sur ces communautés pour fonder un pouvoir de type nouveau, qui est éminemment souverain. À cela, le "Transport" aux Valois ne changea rien : il s'inscrivit au contraire dans la continuité de ce qu'avait voulu Humbert II en 1343. Le Statut de 1349, c'est-à-dire la charte concédée à l'ensemble de la principauté à l'occasion du Transport, me semble même être une extension des libertés de 1343 à tout le Dauphiné. Les montagnes de Briançonnais, premier territoire ayant reçu en tant que

44. H. Falque-Vert, Les hommes et la montagne..., op. cit., p. 439-449.

45. N. Chorier, Histoire..., op. cit. Repris par J. Routier, Les relations avec la Savoie d'une famille de seigneurs briançonnais : les Bardonnèche des Ambrois, s.l.n.d., et par L. des Ambrois de Névache, Notes et souvenirs inédits, Bologne, 1901, p. 290.

46. Voir les hommages qu'ils prêtèrent à I lumbert II en 1334, AD Isère, B 2962, $f^{\circ} 505 v$. Ils étaient de fidèles officiers delphinaux et le demeurèrent : voir A. Lemonde, De la principauté..., op. cit., p. 244-245 et chapitre 2.

47. Ibid. 
L'intégration politique modèle d'un bailliage montagnard 149

tel des libertés, avaient donc servi de terrain d'essai, de la même façon qu'elles avaient servi de point d'ancrage à l'ascension des Albon au $\mathrm{XI}^{c}$ siècle et qu'elles étaient ensuite devenues le lieu par excellence de leur enracinement territorial au XIII siècle. Peut-être est-ce donc dans ce caractère moteur à l'intégration politique, non démenti à travers les siècles, qu'il faut lire une véritable spécificité montagnarde?

Au bout du compte en tout cas, dans la deuxième moitié du $\mathrm{XIV}^{\varepsilon}$ siècle, ces montagnes sont gouvernées en profondeur, en dépit de leur extrême pauvreté, de leur éloignement des grands centres du pouvoir, de leur difficulté d'accès. Sur ce plan-là, les Valois agirent en pleine continuité : ils étaient seulement beaucoup plus riches et purent s'offrir les services d'un artiste pour dresser, en 1422, ce tableau de la Varaita à l'occasion d'un conflit de bornage avec le marquis de Saluces ${ }^{48}$. Commandités par le procureur fiscal, ces tableaux représentent un bel aboutissement de la conquête de la montagne comme territoire par le pouvoir : un territoire limité par une frontière comme il se doit, c'est le but de l'œuvre, mais aussi territoire habité, avec ses maisons de village et d'alpages, ses champs et ses prés, son église et son château, etc. Les inaccessibles sommets eux aussi semblent gouvernés : c'est du moins ce que leur représentation suggestive paraît évoquer. 